\title{
Acetylcholinesterase Enhances Neurite Growth and Synapse Development through Alternative Contributions of Its Hydrolytic Capacity, Core Protein, and Variable C Termini
}

\author{
Meira Sternfeld, ${ }^{1}$ Guo-li Ming, ${ }^{2}$ Hong-jun Song, ${ }^{2}$ Keren Sela, ${ }^{1}$ Rina Timberg, ${ }^{1}$ Mu-ming Poo, ${ }^{2}$ and \\ Hermona Soreq ${ }^{1}$ \\ 1Department of Biological Chemistry, The Life Sciences Institute, The Hebrew University of Jerusalem, 91904, Israel, and \\ 2Department of Biology, University of California at San Diego, La Jolla, California 92093-0357
}

\begin{abstract}
Accumulated indirect evidence suggests nerve growthpromoting activities for acetylcholinesterase (AChE). To determine unequivocally whether such activities exist, whether they are related to the capacities of this enzyme to hydrolyze acetylcholine and enhance synapse development, and whether they are associated with alternative splicing variants of AChEmRNA, we used four recombinant human AChEDNA vectors. When Xenopus laevis embryos were injected with a vector expressing the synapse-characteristic human AChE-E6, which contains the exon 6 -encoded $\mathrm{C}$ terminus, cultured spinal neurons expressing this enzyme grew threefold faster than cocultured control neurons. Similar enhancement occurred in neurons expressing an insertion-inactivated human AChEE6-IN protein, containing the same $\mathrm{C}$ terminus, and displaying indistinguishable immunochemical and electrophoretic migration properties from AChE-E6, but incapable of hydrolyzing acetylcholine. In contrast, the nonsynaptic secretory human AChE-14, which contains the pseudointron 4-derived C terminus, did not affect neurite growth. Moreover, no growth promo-
\end{abstract}

tion occurred in neurons expressing the catalytically active C-terminally truncated human AChE-E4, demonstrating a dominant role for the E6-derived $C$ terminus in neurite extension. Also, AChE-E6 was the only active enzyme variant to be associated with Xenopus membranes. However, postsynaptic length measurements demonstrated that both AChE-E6 and AChE-E4 enhanced the development of neuromuscular junctions in vivo, unlike the catalytically inert AChE-E6-IN and the nonsynaptic AChE-I4. These findings demonstrate an evolutionarily conserved synaptogenic activity for AChE that depends on its hydrolytic capacity but not on its membrane association. Moreover, this synaptogenic effect differs from the growth-promoting activity of AChE, which is unrelated to its hydrolytic capacity yet depends on its exon 6-mediated membrane association.

Key words: acetylcholinesterase; alternative C termini; neurogenesis; neurite extension; noncatalytic function; Xenopus spinal neurons; synaptogenesis; neuromuscular junctions
Acetylcholinesterase (AChE) hydrolyzes the neurotransmitter acetylcholine $(\mathrm{ACh})$ released from nerve terminals at neuromuscular junctions (NMJs) and brain cholinergic synapses, thus terminating synaptic transmission (Salpeter, 1967). Potential noncatalytic functions of $\mathrm{AChE}$ were implicated by findings that certain AChE inhibitors decrease chick neurite outgrowth in culture and that externally added AChE stimulates this process regardless of the presence of specific inhibitors (Layer et al., 1993; Jones et al., 1995; Small et al., 1995). Sequence homology between AChE and several adhesion molecules (de La Escalera et al., 1990; Auld et al., 1995; Ichtchenko et al., 1995) and the early appearance of AChE in developing embryos before the onset of cholinergic neurotransmission (Layer and Willbold, 1995) also suggest that AChE may play a developmental function in cellular development and neuronal growth that is unrelated to its classic

Received Oct. 2, 1997; revised Nov. 21, 1997; accepted Nov. 26, 1997.

This work was supported by grants from National Institutes of Health (NS 31923) to M-m.P., and U.S. Army Medical Research and Development Command (DAMD 17-97-1-7007), the Israeli Ministry of Defense, and the Binational Science Foundation United States-Israel (96/00110/1) to H.S. We thank Dr. U. Brodbeck, Bern, Switzerland, for anti-AChE antibodies, and Ms. Daniela Kaufer for help with experiments.

M.S. and G.M. contributed equally to this work.

Correspondence should be addressed to Hermona Soreq, Department of Biological Chemistry, The Life Sciences Institute, The Hebrew University of Jerusalem, 91904, Israel.

Copyright (C) 1998 Society for Neuroscience $\quad 0270-6474 / 98 / 181240-10 \$ 05.00 / 0$
ACh hydrolyzing activity. However, experiments aimed at the noncatalytic nature of the neurogenic activity of AChE were all based on the indirect use of inhibitors or involved external addition of AChE to the culture medium (Jones et al., 1995) or solid substrate (Layer et al., 1993; Small et al., 1995). This called for studies in which the activity levels of AChE would be changed within the tested neurons themselves.

Human pre-AChEmRNA may be alternatively spliced at its $3^{\prime}$ end to yield three mature AChEmRNAs encoding protein products with three distinct C termini (Ben Aziz-Aloya et al., 1993; Karpel et al., 1994). These include the brain-abundant exon 6-encoded C-terminal peptide, the hematopoietic exon 5-encoded $\mathrm{C}$ terminus, which enables glycophospholipid attachment, and the $\mathrm{C}$ terminus derived from the open reading frame of the tumorabundant pseudointron 4. The brain and muscle human (h) AChE form (hAChE-E6), expressed in developing Xenopus laevis embryos, accumulates in and enlarges the postsynaptic length of neuromuscular junctions (NMJs) (Seidman et al., 1994; 1995). Transgenic mice expressing hAChE-E6 show NMJ enlargement and late onset neuromotor deterioration (Andres et al., 1997). In contrast, DNA encoding the read-through form of hAChEmRNA (ACHE-I4/E5) causes production and secretion of an enzyme C terminated by the I4-encoded peptide (hAChE-I4) in ciliated and secretory epidermal cells of Xenopus embryos. Moreover, hAChE-I4 did not reach NMJs or affect their length (Seidman et 
al., 1995). When transfected into glioma cells, ACHE-I4/E5 caused the appearance of small, processless round cells, whereas hAChE-E6 transfection induced process extension (Karpel et al., 1996). To explore the involvement of the catalytic activity and the alternative $\mathrm{C}$ termini of $\mathrm{AChE}$ in its neurogenic or synaptogenic activities, we constructed two novel hAChEDNA vectors. One of these encodes a truncated form of the enzyme, devoid of any of the natural $\mathrm{C}$ termini; the other encodes an insert-disrupted form of the enzyme, incapable of hydrolyzing ACh yet recognized by anti-AChE antibodies. These two constructs and the above hACHE-E6 and hACHE-I4/E5 DNAs were microinjected into Xenopus oocytes and embryos. The biochemical and hydrodynamic properties of the resultant proteins were then compared with the effects of each of these $\mathrm{AChE}$ variants on neurite extension from spinal neurons and on in vivo NMJ development in Xenopus.

\section{MATERIALS AND METHODS}

Construction of vectors. The plasmids referred to here as ACHE-E6 and ACHE-I4/E5 have been described in detail (Ben Aziz-Aloya et al., 1993; Seidman et al., 1995). To create a DNA construct encoding a truncated form of hAChE, lacking either of the native $\mathrm{C}$ termini, we used a two-phase PCR engineering procedure (Higuchi, 1990) using the recombinant human ACHE cDNA and genomic clones (Soreq et al., 1990). In the first PCR phase, performed essentially as described (Karpel et al., 1994), ACHE-E6 served as template. Two partially overlapping products were produced in which ACHE exon 4 and the SV40 polyadenylation signal were joined together, using primers containing the overlapping sequence: E3/1522+ 5'-CGGCTCTACGCCTACGTCTTTGAACAC CGTGCTTC-3'; E4del4-5'-TAACGTCGACTATCAGGTGGCGCTGA GCAATTTGGGGG-3'; E4del3 + 5'-TTGCTCAGCGCCACCTGATAGT CGACGTTAACTTGTTTATTGCAGCTTATAATGG-3'; SV40 PolyA5'AtgatTTGgaCAaACCACAACTAGAATGCAGTG-3'. Primers were named according to their position in the human ACHE alternative sequences and vectors. After removal of the primers, the two products were combined into one longer product by a second PCR reaction in which they served as templates. External primers E3/1522+ and SV40 polyA - were used in the second phase to create a fragment consisting of the $3^{\prime}$ end of exon 3 , exon 4 , and the polyA signal. PCR reaction was as above except that in the first cycle the denaturing step was at $94^{\circ} \mathrm{C}$ for 5 min and the annealing step was from 94 to $50^{\circ} \mathrm{C}$ (slope rate of $1^{\circ} \mathrm{C}$ per 30 $\mathrm{sec}$ ). The product and the original ACHE-E6 plasmid were restricted using enzymes NotI and SalI, and the two products were then ligated.

To construct the disrupted ACHE coding sequence we inserted an in-frame sequence of 21 nucleotides, six bases downstream of the codon for the active site serine, located in exon 2. The technique used was the same two-phase PCR described above. In the first PCR phase we used primers containing the inserted sequence. Primers used were E2/340+ 5'-GCTTTCCTGGGCATCCCCTTTGCGGAGCCA-3'; E2ins2-5' TCCaccgaattgaggatgtcgecacgcgetCTCCCCAAACAGCGT-3'; E2ins1+ 5' - AGCGCGtggcgacatcctcaattcggtggaGCCGCCTCGGCGGGCAT-3'; 1212-5'-GAAGTCTCCCGCGTTGATCAGGGCCTCTGG-3'. The inserted sequence is designated by lower case letters. Primers E2/340+ and E2ins2- were used to link the inserted sequence to the upstream PCR product, and primers E2ins1+ and E2/1212- were used to link it to the downstream product. The second PCR phase was performed using external primers E2/340+ and E2/1212-. Stage II PCR products and ACHE-E6 were restricted using enzymes BstEII and $S p h \mathrm{I}$ and ligated. First and second phase PCR reactions were as above. After construction, the accuracy and integrity of both constructs was validated by DNA sequencing.

Recombinant AChE production and assays of hydrolytic activity. Xenopus oocytes were microinjected with $10 \mathrm{ng}$ of DNA of each recombinant AChE plasmid, incubated for $48 \mathrm{hr}$, and homogenized in high-saltdetergent buffer as described previously (Neville et al., 1990). Homogenates were frozen until use. AChE activity was measured by evaluating acetylthiocholine (ATCh) hydrolysis using 96-well microtiter plates. $\mathrm{pH}$ dependence of AChE activity was assessed using phosphate buffer at the $\mathrm{pH}$ range 5.8-8.0, with intervals of 0.2. For $K_{\mathrm{m}}$ and substrate inhibition experiments, we used ATCh in the concentration range of $0.05-60 \mathrm{~mm}$ and the GraFit 3.0 program (Erithacus Software limited, Staines, UK).
For enzyme stability studies, oocyte homogenates were incubated at $19-42^{\circ} \mathrm{C}$ for $0-5 \mathrm{hr}$, after which AChE catalytic activities in each of the homogenates were assessed as above.

Xenopus embryo microinjection and subcellular fractionation. In vitro fertilization of mature Xenopus eggs and blastomere microinjection were performed as described elsewhere (Seidman et al., 1994), except that embryos were raised in $19-21^{\circ} \mathrm{C}$. Subcellular fractionation of 1-, 2-, and 3-d-old embryos into low-salt (0.01 м Tris-HCl, $\mathrm{pH} 7.4,0.05 \mathrm{M} \mathrm{MgCl}_{2}, 144$ $\mathrm{mm} \mathrm{NaCl})$, low-salt-detergent ( $1 \%$ Triton $\mathrm{X}-100$ in $0.01 \mathrm{~m}$ sodium phosphate, $\mathrm{pH} 7.4)$, and high-salt ( $1 \mathrm{M} \mathrm{NaCl}$ in $0.01 \mathrm{~m}$ sodium phosphate, $\mathrm{pH}$ 7.4) buffers was performed as described previously (Seidman et al., 1994).

Protein blot analyses and immunocytochemistry. Denaturing SDSPAGE and blotting were essentially as described elsewhere (Seidman et al., 1994), except that after transfer the blots were washed with $1 \times$ PBS (80 mM NaH $\mathrm{PO}_{4}, 20 \mathrm{~mm} \mathrm{Na} \mathrm{HPO}_{4}, \mathrm{pH} 7.4$ ), 0.5\% Tween-20, $18 \%$ glucose, $10 \%$ glycerol, $2.5 \%$ bovine serum albumin, and $1 \%$ skim milk. Immunodetection was performed using a pool of monoclonal antibodies $(132-1,2,3 ; 6 \mu \mathrm{g} / \mathrm{ml}$ each) raised against denatured human brain AChE, and a 1:2 $\times 10^{4}$ dilution of a horseradish-peroxidase-conjugated sheep anti-mouse IgG (Jackson Laboratories, Bar Harbor, ME). Chemiluminescent detection was performed with the ECL kit (Amersham Life Sciences) as instructed. Ten microliter samples of oocyte homogenate (equivalent to $\sim 50 \mathrm{ng} \mathrm{AChE}$ ) were loaded on each lane. For enzyme activity blots, we used nondenaturing gel electrophoresis followed by incubation in ATCh staining mixture (Seidman et al., 1995), using similar amounts of oocyte homogenates.

For immunochemical AChE detection in situ, cells were fixed with $2 \%$ paraformaldehyde in PBS for $30 \mathrm{~min}$ at room temperature and then permeabilized with $0.1 \%$ Triton X-100 (20 min). After they were washed with TBST (10 mM Tris-HCl, pH 7.0, $150 \mathrm{~mm} \mathrm{NaCl,} \mathrm{0.05 \%} \mathrm{Tween-20),}$ cells were incubated with anti-human AChE antibodies (mouse monoclonal antibody $132-1 ; 1: 1000$ dilution in TBST containing 10\% normal goat serum) at $4^{\circ} \mathrm{C}$ overnight. After washes with TBST $(5 \times 30 \mathrm{~min})$, cells were incubated with goat anti-mouse $\mathrm{IgG}$ conjugated to fluorescein (Sigma) (1:100 in TBST containing $10 \%$ normal goat serum) at $22^{\circ} \mathrm{C}$ for $2 \mathrm{hr}$. After the same washing procedure as described above, cells were mounted and observed under a Nikon Diaphot microscope with a $20 \times /$ 1.25 objective.

Culture preparation. Xenopus nerve-muscle cultures were prepared according to previously reported methods (Spitzer and Lamborghini, 1976; Anderson et al., 1977; Tabti and Poo, 1995). Briefly, neural tubes and the associated myotomal tissue of 1-d-old Xenopus embryos (stage 20-23 according to Nieuwkoop and Faber, 1967) were dissociated in $\mathrm{Ca}^{2+}-\mathrm{Mg}^{2+}$-free saline supplemented with EDTA (115 mM NaCl, 2.6 $\mathrm{mm} \mathrm{KCl,} 10 \mathrm{~mm}$ HEPES, 0.4 mM EDTA, pH 7.6) for 15-20 min. The cells were plated on glass coverslips and used for experiments after $6 \mathrm{hr}$ of incubation at room temperature. The culture medium consisted (vol/ vol) of $49 \%$ Leibovitz L-15 medium (Life Technologies, Gaithersburg, MD), $1 \%$ bovine serum (Life Technologies), and $50 \%$ Ringer's solution (115 mm NaCl, $2 \mathrm{~mm} \mathrm{CaCl}_{2}, 2.6 \mathrm{~mm} \mathrm{KCl,} 10 \mathrm{~mm}$ HEPES, pH 7.6).

Neurite length measurements. Line drawings of isolated neurons and their neuritic processes were traced from the video monitor display of recorded microscopic images. The tip of the growing neurite was defined as the distal leading edge of the phase-dark palm of the growth cone, without considering the filopodial extension. The entire trajectory of the neurite, including all of its branches, was measured with a digitizing pad (Houston Instruments), and the total length of each neurite was calculated by a microcomputer. The rate of extension (micrometers per hour) was determined by dividing the net increase in neurite length (in micrometers) by the duration of observation (in hours).

Electron microscopy and morphometric analyses. Histochemical staining, transmission electron microscopy, and morphometric analyses were performed as described previously (Ben Aziz-Aloya et al., 1993; Seidman et al., 1995).

\section{RESULTS}

\section{Construction and analyses of hAChE variants}

To delineate functions and domains of $\mathrm{AChE}$ variants that are involved in neuron growth and synaptogenesis, two novel hAChEDNA vectors were constructed by a two-phase PCR procedure (see Materials and Methods). One of these constructs, ACHE-E4 (Fig. $1 A$ ), carries a truncated coding sequence containing exons 2 , 

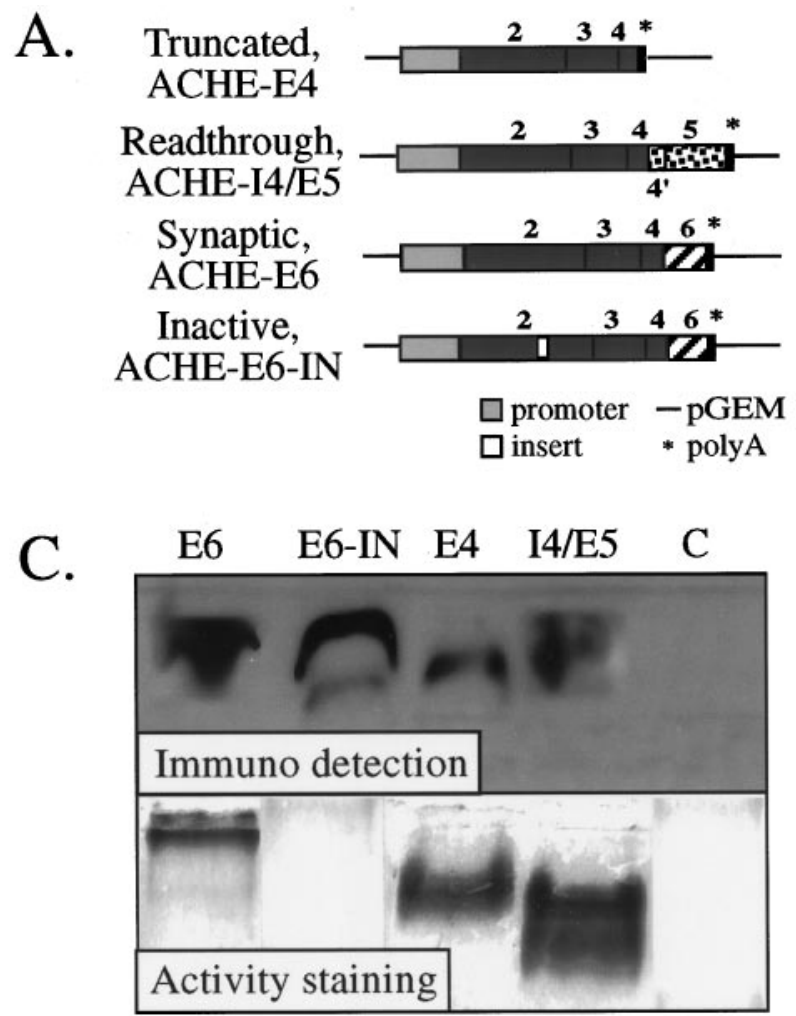
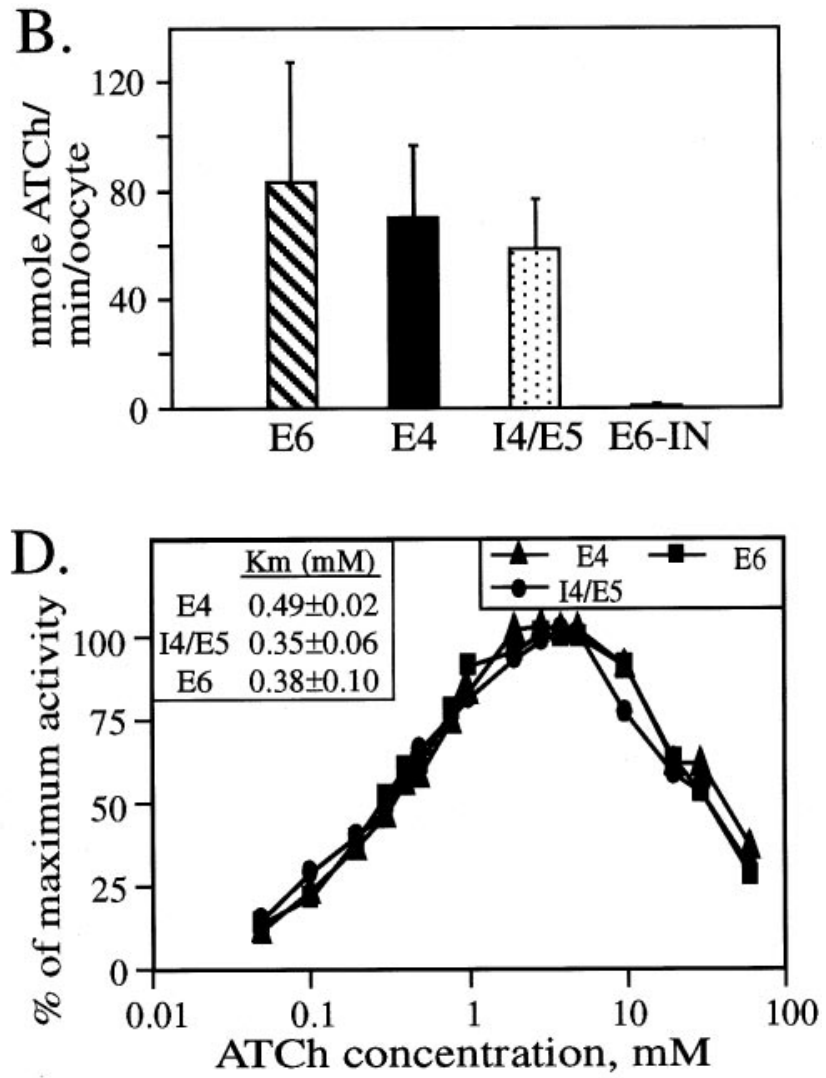

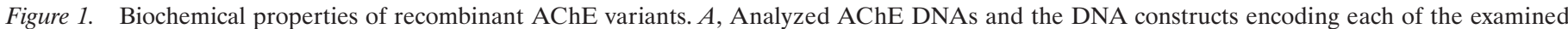

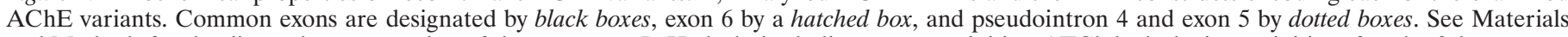

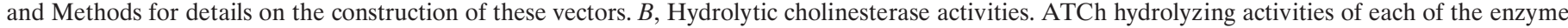

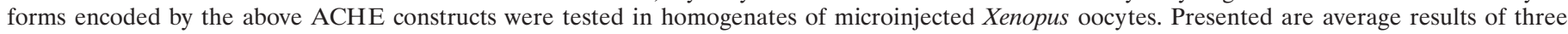

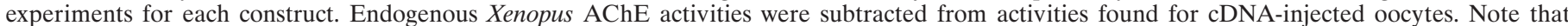

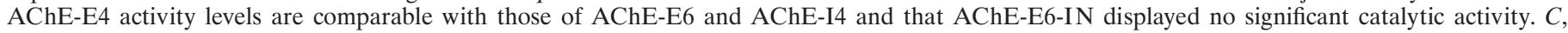

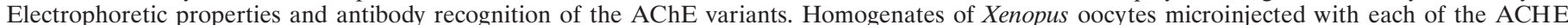

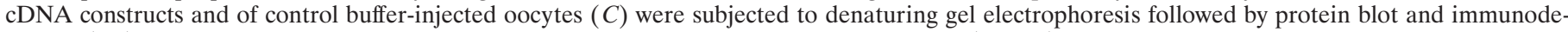

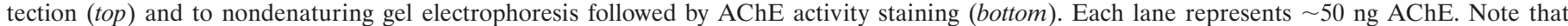

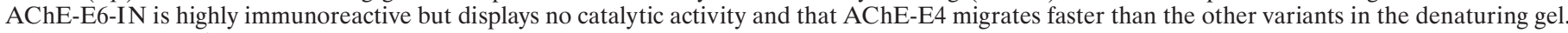

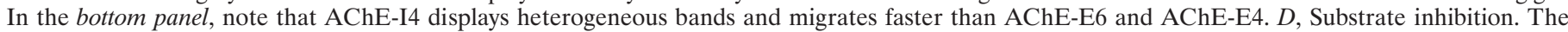

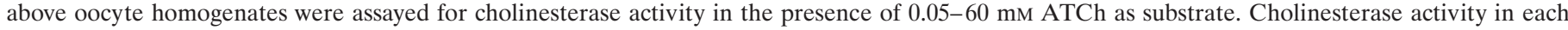

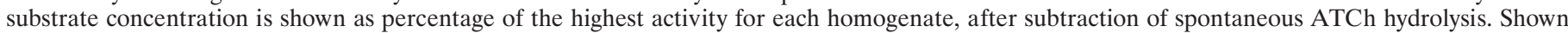
is one representative of two experiments. Inset, $K_{\mathrm{m}}$ values of the recombinant hAChE variants.

3, and 4 of the human ACHE gene. The other construct, ACHEE6-IN (Fig. $1 A$ ), encodes a protein identical to the synapseaccumulating AChE-E6, except that it carries an in-frame insert of seven amino acids near the active site protein sequence, which should render it inactive. Similar to the previously used ACHE-E6 (Ben Aziz-Aloya et al., 1993) (Fig. 1A) and ACHEI4/E5 (Seidman et al., 1995) (Fig. $1 A$ ), transcription of both these constructs was regulated by the cytomegalovirus promoter, and they both contain the SV40 polyadenylation signal. Together, this set of four constructs enabled us to explore the biochemical and morphogenic activities of AChEs with three distinct $\mathrm{C}$ termini (encoded by E6, I4, or E4) and of the synaptic AChE-E6 enzyme with or without catalytic capacity.

For biochemical characterization of their protein products, all four plasmids were microinjected into Xenopus oocytes. The catalytic activity of the resultant proteins was then assessed by measuring the hydrolysis rate of acetylthiocholine (ATCh) in oocyte homogenates. As predicted, oocytes expressing the disrupted form, ACHE-E6-IN, displayed exceedingly low activity levels (Fig. $1 B$ ), which were similar to those of the two experi- mental controls: buffer-injected and uninjected oocytes (not shown), most probably reflecting the endogenous Xenopus AChE activity levels. As expected, both natural AChE variants, terminated with the E6-encoded $\mathrm{C}$ terminus or with that encoded by I4, showed high activity levels (Fig. $1 B$ ), confirming previous results (Schwarz et al., 1995a; Seidman et al., 1995). The novel truncated form of AChE, encoded by ACHE-E4, displayed activity levels within the range of the other two variants (Fig. $1 B$ ). This demonstrated that neither of the natural $\mathrm{C}$ termini encoded by E6 or I4 is essential for the ACh hydrolytic activity of AChE.

\section{Electrophoretic distinctions and hydrolytic similarities}

In consideration of the possibility that the low activity levels observed for the ACHE-E6-IN homogenates did not reflect inactivation but were caused by impaired production of this protein in oocytes, we subjected the various recombinant hAChEs to denaturing gel electrophoresis followed by immunoblotting. Selective immunodetection of hAChE bands (Fig. 1C, top panel) demonstrated that the Xenopus system is capable of producing AChE-E6-IN in size and amounts comparable to those of the 
other AChE variants. The electrophoretic migration distance of all variants except AChE-E4 matched the expected molecular weight of $66 \mathrm{kDa}$, whereas AChE-E4 migrated somewhat faster, consistent with its truncated $\mathrm{C}$ terminus. Minor amounts of an immunopositive protein, with a migration distance similar to that of AChE-E4, could also be seen in the lanes loaded with the protein products of ACHE-E6 and ACHE-E6-IN (Fig. 1C, top panel). Catalytic activity and intact $\mathrm{C}$ termini are therefore not obligatory requirements for production and stability of this protein in the Xenopus milieu.

When subjected to nondenaturing gel electrophoresis followed by AChE activity staining, the enzyme produced by ACHEE6-IN showed no detectable catalytic activity, as opposed to the other variants, which were all highly active (Fig. $1 C$, bottom panel). The migration of AChE-E6 was considerably slower and the band was much sharper than those of AChE-I4, which displayed faster migrating heterogeneous bands. AChE-E4 showed an intermediate band. The four tested hAChE variants thus differed in their Stokes radius and charge and active site conformation, whereas they maintained similar primary folding and production efficiency.

Having shown that ACHE-E6, ACHE-I4/E5, and ACHE-E4 all encode for active enzymes, we wished to examine whether changing the $\mathrm{C}$ terminus of $\mathrm{AChE}$ did not affect its catalytic properties in a more subtle manner. Interestingly, the Xenopus enzyme retained its full catalytic activity after $5 \mathrm{hr}$ at $42^{\circ} \mathrm{C}$, whereas all hAChE variants lost $50 \%$ of their activity (not shown). However, all three hAChE variants were found to have $K_{\mathrm{m}}$ values within the same range of the previously reported value (0.3 mu for AChE-E6 in Xenopus oocytes) (Seidman et al., 1994) and were similarly inhibited by high substrate concentrations (Fig. $1 D)$. Also, substrate hydrolysis by all AChE forms was similarly enhanced within the $\mathrm{pH}$ range of 5.8-8.0 (data not shown), in agreement with reports of others (for review, see Schwarz et al., 1995b). There was therefore no indication whatsoever for involvement of the variable $\mathrm{C}$ termini of $\mathrm{AChE}$ in its catalytic properties, consistent with previous reports in which enzymatic cleavage of the $\mathrm{C}$ terminus was used to obtain a homogeneous catalytically active AChE preparation for x-ray diffraction analysis (Sussman et al., 1991).

\section{Expression of human AChE in Xenopus spinal neurons}

Expression of hAChE in Xenopus embryonic neurons was examined after injection of each of the above hAChEDNA constructs into one of the blastomeres of two-cell stage Xenopus embryos. To facilitate identification of living neurons expressing hAChE during neurite growth assays, fluorescent dextran was co-injected with the DNA. The progeny neurons of the injected blastomere could then be identified by the presence of fluorescent dextran. Confirmation of $\mathrm{AChE}$ expression in individual spinal neurons was then obtained by immunocytochemical staining of the dissociated neurons from 1-d-old embryos, using monoclonal antibodies specific for hAChE (Seidman et al., 1995). The reliability of fluorescent dextran as a marker for neurons overexpressing AChE was examined in the following experiment. Nerve-muscle cultures were prepared from embryos injected with ACHE-E6 cDNA and rhodamine-dextran. Dextran-positive neurons in 1-dold cultures were identified and recorded. The same cultures were then processed for immunocytochemical staining with antibodies against AChE. In control cultures prepared from embryos not injected with AChE-E6 cDNA, there was a negligible level of AChE staining. In cultures prepared from AChE-E6 cDNA and dextran-injected embryos, we found that $92 \%$ (36 of 39 cells) of dextran-positive neurons exhibited AChE staining, whereas $95 \%$ (55 of 58 cells) of dextran-negative neurons exhibited undetectable AChE staining. Figure 2 depicts examples of (AChE+) and $(\mathrm{AChE}-)$ neurons, together with their bright-field images at 7, 8, and $9 \mathrm{hr}$ in culture, before the staining of AChE. In this culture, dextran-positive muscle cells also showed, as expected, elevated staining with AChE. Thus, immunostaining for hAChE shows that hAChE-E6 is expressed in these spinal neurons and that dextran fluorescence is a reliable marker for AChE expression.

\section{Effects of expressing human AChE on neurite growth}

Six hours after plating of dissociated Xenopus neural tube cells in culture, many spinal neurons exhibit active neurite outgrowth. Only isolated neurons not in contact with any other cell were used in this study, on which two types of neurite growth assays were made. First, the total neurite length of each neuron in the culture was measured. Second, extension of individual neurites was measured for a 3-4 hr period at $1 \mathrm{hr}$ intervals (from 6 to $10 \mathrm{hr}$ after plating). The hAChE-expressing (AChE+) neurons were identified by the presence of fluorescent dextran in the cell. To reduce culture-to-culture variation, similar numbers of $(\mathrm{AChE}+)$ and control neurons in the same culture or cultures from the same batch of embryos were examined. This analysis revealed that the average neurite length (the entire trajectory of the neurite, including all its branches) was $124.0 \pm 11.7 \mu \mathrm{m}(\mathrm{SEM} ; n=32)$ in (AChE-E6+)-expressing neurons, which is significantly longer ( $p<0.001$; two-tailed $t$ test) than that observed for noninjected neurons $(88.2 \pm 10.3 \mu \mathrm{m}$; SEM; $n=33)$ or AChE-I4-expressing neurons $(77.5 \pm 8.2 \mu \mathrm{m}$; SEM; $n=27)$. To illustrate the overall difference in neurite growth for a large number of neurons, composite drawings were made by superimposing tracings of the video images of randomly chosen neurons, 15 from each of the noninjected control, (AChE-E6+), and (AChE-I4+) groups, respectively (Fig. 3). It is clear from this figure that the net neurite extension over the first $9 \mathrm{hr}$ in culture was substantially longer in (AChE-E6+) neurons.

The higher total neurite length at a particular time in culture may reflect a higher growth rate of each neurite, an earlier onset of neurite outgrowth from the soma after cell plating, or both. Direct measurements of the growth rate of individual neurites during the 6-10 hr period revealed that (AChE-E6+) neurites indeed exhibit a higher growth rate. As shown in Table 1, in the same group of cultures, the average growth rate was $12.9 \pm 2.7$ $\mu \mathrm{m} / \mathrm{hr}$ (SEM; $n=33$ ) for noninjected control neurons but was elevated to $37.9 \pm 5.2 \mu \mathrm{m} / \mathrm{hr}(\mathrm{SEM} ; n=32)$ for $(\mathrm{AChE}-\mathrm{E} 6+)$ neurons, the difference being highly significant $(p<0.005$; twotailed $t$ test). In contrast, neurons expressing the read-through form AChE-I4 did not show any difference in growth rate compared with the noninjected controls. The enhanced growth rate of $(\mathrm{AChE}-\mathrm{E} 6+)$ neurons was sufficient to account for the difference in the overall neurite length described above, although earlier neurite initiation could also have occurred. In addition to the growth rate, we also measured the number of neuritic branches in each group of neurons. As shown in Table 1, no significant difference was found between any of the groups.

\section{Growth promotion is unrelated to $\mathrm{ACh}$ hydrolytic activity}

Whether the growth-promoting effect on Xenopus neurons was partially or entirely caused by the catalytic activity of the enzyme in hydrolyzing ACh was tested by expressing AChE-E6-IN, the 


\section{7 hour 8 hour}
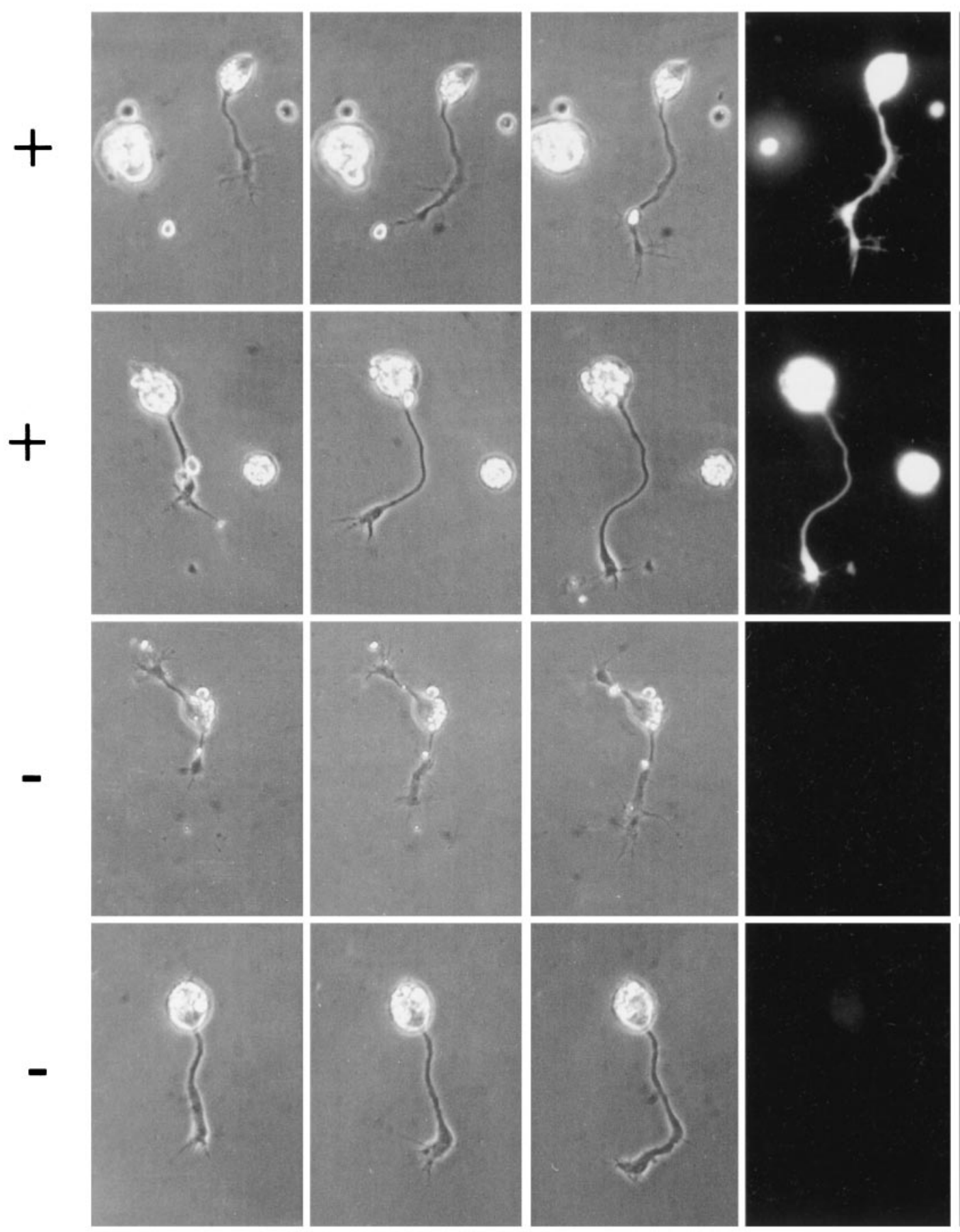
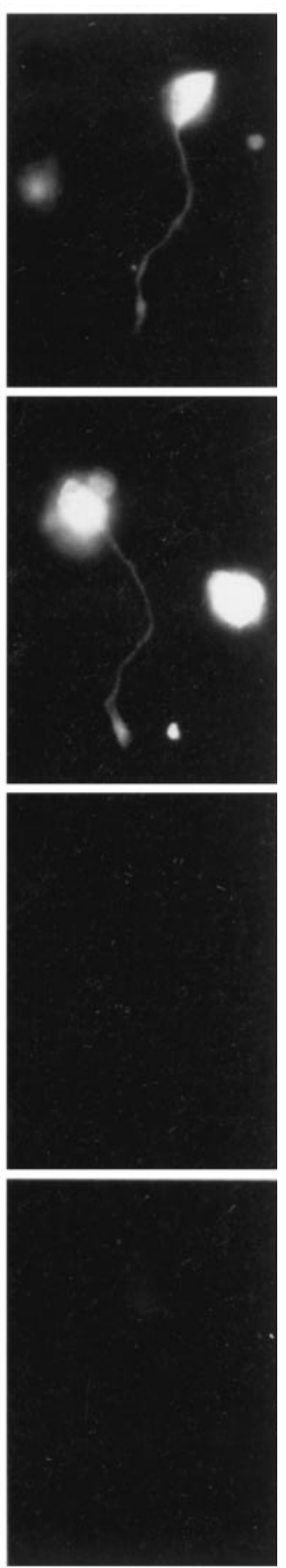

Figure 2. Neurons expressing human AChE-E6 (+) and control neurons (-) in Xenopus cultures. Xenopus embryos were co-injected with AChE-E6 DNA and rhodamine-dextran complexes, and their spinal neurons were dissociated into culture $1 \mathrm{~d}$ later. Bright-field images were taken at 7,8 , and $9 \mathrm{hr}$ after cell plating. Both the total neurite length and the rate of neurite growth were measured during this period. Fluorescence micrographs on the right of the $9 \mathrm{hr}$ photographs depict the rhodamine fluorescence of dextran complexes, which were co-injected with the cDNA. Indirect fluorescein immunofluorescence staining of AChE observed at the end of the experiment is shown on the last right panel. Note the correlation between dextran fluorescence and AChE staining. Staining and imaging conditions were identical for all four cells, which were from the same culture. Fluorescently labeled cells $(+)$ were positive with both red and green filters, whereas negative $(-)$ cells remained invisible in both. Scale bar, $20 \mu \mathrm{m}$. 


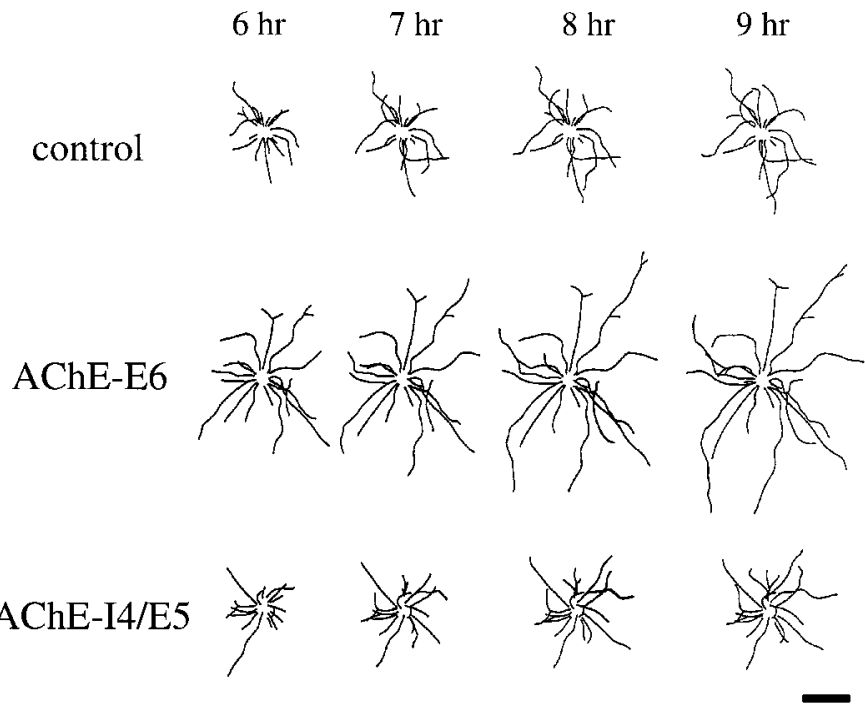

Figure 3. Effects of expressing human AChE on the growth of Xenopus spinal neurons. Composite concentric line drawings were made from video images of 12 isolated spinal neurons at $6,7,8$, and $9 \mathrm{hr}$ after cell plating. The center of the neuronal soma (deleted from this image) was placed in the center of each drawing. Note consistent overall neurite length promotion in neurons that expressed AChE-E6 but not AChE-I4 Neurons derived from uninjected blastomeres served as controls. Scale bar, $20 \mu \mathrm{m}$.

insertion-inactivated form of AChE-E6, which lacks ACh hydrolytic activity (see above). As shown in Table 1, (AChE-E6+) and (ACHE-E6-IN+) neurites were similar both in the total neurite length after 9-10 hr in culture and in the rate of neurite growth during this period. Thus, the ability of hAChE to promote growth was associated with the presence of the E6-derived $\mathrm{C}$ terminus, regardless of the catalytic activity.

\section{The E6-derived $\mathbf{C}$ terminus is essential for growth promotion}

The ability of AChE-E6 but not AChE-I4 to promote neurite growth could be attributable to a dominant-positive effect of the AChE-E6 C terminus (conferred by a sequence and/or structural element present in this domain), or to a dominant-negative effect exerted by the I4-encoded $\mathrm{C}$ terminus (i.e., the I4 peptide could interfere with the growth-promoting properties of other domain(s) in the AChE core protein). This problem was examined by expressing the truncated AChE-E4 form of AChE, which retains the ACh hydrolytic activity yet lacks either the E6- or the I4-derived natural C-terminal peptides. The inability of AChE-E4 to promote growth (Table 1) proved the first option correct. Therefore, growth promotion of Xenopus spinal neurons by $\mathrm{AChE}$ did not require ACh hydrolytic activity; neither was it affected by the presence or absence of the I4-derived $\mathrm{C}$ terminus. Rather, to exert this growth-promoting activity the E6-derived synapse-characteristic $\mathrm{C}$ terminus must be present.

\section{The growth promotion activity of AChE is associated with membrane interaction}

Despite their sequence and biochemical similarities, AChE-E6 and AChE-I4 have previously been shown to be differentially localized in Xenopus embryos, both intra- and intercellularly (Seidman et al., 1995). AChE-E6 was found to be associated with NMJs and AChE-I4 localized in epidermis and secreted therefrom. To compare the hydrodynamic and membrane association properties of the different recombinant hAChE variants in relation to their growth-promoting capacities, we microinjected ACHE-E4, ACHE-E6, or ACHE-I4/E5 DNAs into in vitro fertilized Xenopus eggs. Sequential extractions of injected embryos into low-salt, low-salt-detergent, and high-salt buffers yielded hAChE-containing homogenates from 1-, 2-, and 3-d-old injected Xenopus embryos. AChE catalytic activities measured in these homogenates ranged 2- to 10-fold higher than those of homogenates from uninjected embryos (data not shown) (Seidman et al., 1995). Most importantly, the recombinant hAChE variants differed in their membrane association. Catalytically active AChE in homogenates from ACHE-E4-injected embryos was 92-95\% soluble in low-salt buffer, and AChE-I4 was 74-91\% low-salt soluble (Fig. 4), as compared with 33-53\% for low-salt-soluble AChE-E6. A major fraction $(20-50 \%)$ of hAChE-E6, but no other active variant, partitioned into the low-salt-detergent fraction, reconfirming our previous reports (Seidman et al., 1995) and resembling the solubility pattern of the endogenous Xenopus enzyme (Fig. 4). Thus, although AChE-E6 could be membraneassociated, AChE-E4 and AChE-I4 appear to be soluble proteins that could be secreted from the cells expressing them. Moreover, in all cases except AChE-E4, but including the endogenous $\mathrm{Xe}$ nopus enzyme, there seemed to be a shift in solubility with development, from the low-salt fraction to the detergent and high-salt fractions. This shift may reflect a progressive increase in membrane interaction and association with other components, such as the extracellular matrix, at the time these components are being formed and neurons extend their neurites in vivo.

\section{The synaptogenic and neurite growth-promoting activities of AChE are distinct}

To compare the synaptogenic effect of AChE (Seidman et al., 1995 ) with its neurite growth-promoting capacity, we examined neuromuscular junctions (NMJs) from myotomes of 2-d-old embryos injected with the above four vectors. AChE activity staining (Seidman and Soreq, 1996) followed by transmission electron microscopy was used to assess the in vivo localization of AChE and its synaptogenic activity in Xenopus NMJs. As is apparent from the representative images of NMJs presented in Figure 5, both AChE-E6 and the truncated AChE-E4 accumulated in NMJs in amounts exceeding those in control NMJs. High levels of catalytically active AChE, most likely of endogenous Xenopus origin, were also detected in NMJs from embryos expressing AChE-E6-IN. In contrast, AChE-I4 was absent from NMJs, confirming previous observations (Seidman et al., 1995). These analyses demonstrated that although the E6-encoded $\mathrm{C}$ terminus may promote the interaction of $\mathrm{AChE}$ with $\mathrm{NMJ}$ components, it is not obligatory for NMJ accumulation of catalytically active AChE. Rather, the I4-encoded $\mathrm{C}$ terminus appeared to interfere dominantly with the accumulation of AChE in NMJs. Moreover, postsynaptic length measurements in 2-d-old embryos showed that transgenic expression of both AChE-E6 and the soluble AChE-E4 enlarged NMJs from an average length of $1.82 \pm 0.16$ $\mu \mathrm{m}$ (SEM; $n=55)$ to significantly larger synapses with averages of $2.29 \pm 0.16 \mu \mathrm{m}(\mathrm{SEM} ; n=66)$ and $2.68 \pm 0.36 \mu \mathrm{m}(\mathrm{SEM} ; n=$ $16)$, respectively ( $p<0.05$; two-tailed $t$ test). In contrast, NMJs from embryos expressing AChE-I4 or AChE-E6-IN displayed average postsynaptic lengths that were somewhat smaller yet not significantly different from that of control NMJs $(1.69 \pm 0.19 \mu \mathrm{m}$, SEM, $n=43$, and $1.41 \pm 0.23 \mu \mathrm{m}$, SEM, $n=31$, respectively). The columns in Figure 5 depict this change, which is reflected by a shift in NMJ distribution between two groups: synapses with 
Table 1. Effects of AChE on neurite growth in vitro

\begin{tabular}{|c|c|c|c|c|c|c|}
\hline \multirow[b]{2}{*}{ Construct injected $^{a}$} & & \multicolumn{2}{|c|}{ Total neurite length per cell $(\mu \mathrm{m})$} & \multirow{2}{*}{$\begin{array}{l}\text { Growth rate } \\
(\mu \mathrm{m} / \mathrm{hr})\end{array}$} & \multirow{2}{*}{$\begin{array}{l}\text { Number of } \\
\text { branches }\end{array}$} & \multirow{2}{*}{$\begin{array}{l}\text { Number of cells } \\
\text { (embryos) }\end{array}$} \\
\hline & & at $6-7 \mathrm{hr}$ & at $9-10 \mathrm{hr}$ & & & \\
\hline \multirow[t]{2}{*}{ ACHE-E6 } & + & $64.6 \pm 6.5$ & $124.0 \pm 11.7^{*}$ & $37.9 \pm 5.2^{*}$ & $1.8 \pm 0.1$ & $32(7)$ \\
\hline & - & $55.2 \pm 5.9$ & $88.2 \pm 10.3$ & $12.9 \pm 2.7$ & $1.6 \pm 0.1$ & $33(7)$ \\
\hline \multirow[t]{2}{*}{ ACHE-I4/E5 } & + & $62.0 \pm 10.6$ & $77.5 \pm 8.2$ & $11.6 \pm 2.1$ & $1.7 \pm 0.1$ & $27(8)$ \\
\hline & - & $52.9 \pm 5.0$ & $92.7 \pm 6.2$ & $14.7 \pm 1.9$ & $1.6 \pm 0.1$ & $31(8)$ \\
\hline \multirow[t]{2}{*}{ ACHE-E6-IN } & + & $91.2 \pm 9.8^{*}$ & $156.9 \pm 13.6^{*}$ & $33.6 \pm 3.7^{*}$ & $2.0 \pm 0.1$ & $25(3)$ \\
\hline & - & $47.4 \pm 5.1$ & $62.5 \pm 9.6$ & $11.2 \pm 2.1$ & $1.5 \pm 0.1$ & $28(3)$ \\
\hline \multirow[t]{2}{*}{ ACHE-E4 } & + & $48.0 \pm 7.0$ & $94.2 \pm 9.1$ & $11.9 \pm 3.7$ & $1.7 \pm 0.2$ & $16(4)$ \\
\hline & - & $51.6 \pm 7.4$ & $126.2 \pm 20.4$ & $13.0 \pm 4.2$ & $1.8 \pm 0.2$ & $20(4)$ \\
\hline
\end{tabular}

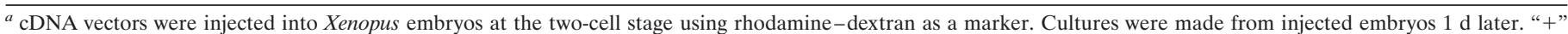
indicates rhodamine-dextran-positive neurons; "-" indicates rhodamine-dextran-negative neurons in the same cultures.

* Significant difference was found between + and - groups (two-tailed $t$ test; $p<0.005$ ).

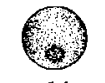

d1
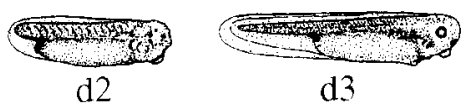

d3
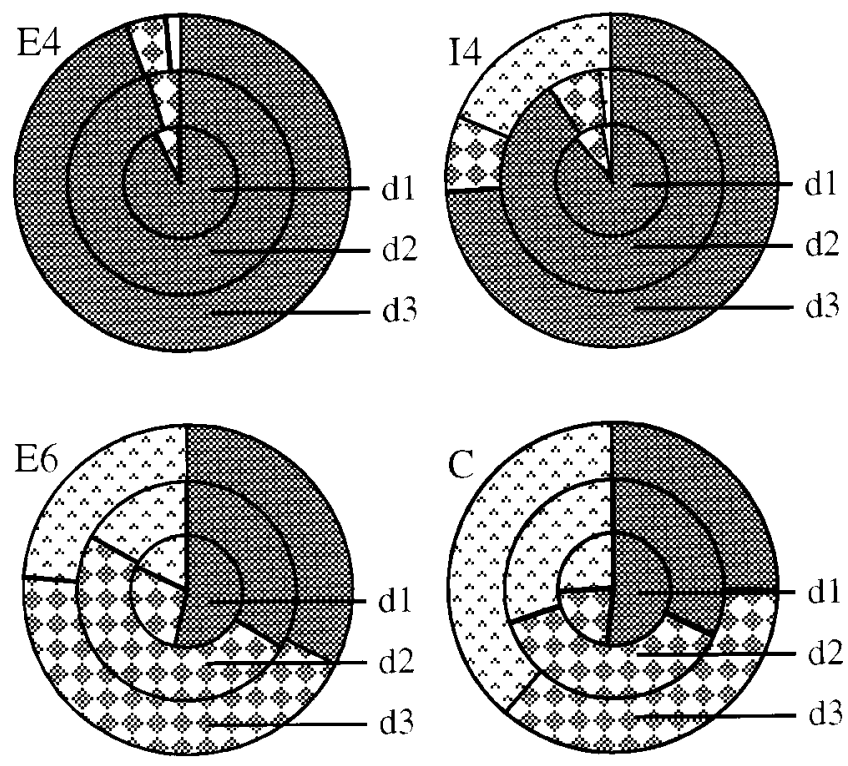
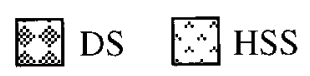

Figure 4. AChE-E6 exhibits developmentally increased membrane association in vivo. Cleaving Xenopus embryos were injected with the various ACHE DNA vectors or with buffer $(C)$, and sequential extractions into low-salt-soluble $(L S S)$, low-salt-detergent-soluble $(D S)$, and high-saltsoluble $(H S S)$ fractions were performed. Endogenous Xenopus AChE activities were subtracted from activities of all other embryo samples. Slices therefore represent the net relative fractions of the total summed activities for the host enzyme and each hAChE variant. Note that AChE-E6 is similar to Xenopus AChE in its lower solubility under low-salt extraction, whereas AChE-E4 and AChE-I4 are both predominantly low-salt soluble. Top, Schematic drawings of 1,- 2-, and 3-d-old Xenopus embryos modeled after those of Deuchar (1966).

postsynaptic lengths smaller or larger than $2.5 \mu \mathrm{m}$. Thus, both the synaptic accumulation and the hydrolytic capacity of the transgenic enzyme were found to be obligatory requirements for its ability to enhance NMJ development. In contrast, the synapto- genic activity of $\mathrm{AChE}$ was found to be unrelated to membrane association, unlike its neuritic growth-promoting function.

\section{DISCUSSION}

We used four recombinant hAChE variants to demonstrate that the neurite growth-promoting activity of $\mathrm{AChE}$ in cultured $\mathrm{Xe}$ nopus neurons depends on the E6-encoded $\mathrm{C}$ terminus but not on catalytic activity, whereas its synaptogenic property in live Xenopus embryos depends both on its ability to accumulate within the synapse and on its hydrolytic capacity. Thus, AChE plays two distinct roles, with different mechanistic requirements, during nervous system development.

That the $\mathrm{C}$ terminus of hAChE modifies the electrophoretic migration properties of the enzyme under both native and denaturing conditions demonstrated that it constitutes an independent domain in the AChE protein, affecting its Stokes radius and surface charge. Human AChE-E6, AChE-I4, and AChE-E4, all of which differ in their $\mathrm{C}$ termini, are catalytically active (this report) and enzymatically indistinguishable (Schwarz et al., 1995a). Cleavage of the E6-encoded C terminus does not affect the catalytic activity of Torpedo AChE (Duval et al., 1992), which demonstrates that the $\mathrm{C}$ terminus is not necessary for substrate hydrolysis in vitro. Our current results further reveal that the intact catalytic activity of AChE in Xenopus laevis is C terminus independent also in vivo. Furthermore, an in-frame insertion of a foreign septapeptide near the active site serine abolished the catalytic activity of the enzyme, consistent with predictions of others (Taylor and Radic, 1994). Neither of these alterations had an apparent effect on the amounts or the immunoreactivity of the enzyme produced in Xenopus oocytes or embryos.

The minor fast-migrating immunoreactive band produced from the hAChE-E6 and hAChE-E6-IN proteins implies partial cleavage of the $\mathrm{C}$ terminus encoded by E6, yielding a protein that is identical to hAChE-E4. The electrophoretic heterogeneity of hAChE-I4 and the fact that part of the hAChE-I4 protein migrated similarly to hAChE-E4 under nondenaturing gel electrophoresis further suggested that the I4-encoded $\mathrm{C}$ terminus could be positioned in several orientations relative to the core domain and be accessible to proteases, consistent with findings of others (Sussman et al., 1991). However, because only minor parts of hAChE-E6 or hAChE-I4 co-migrate with hAChE-E4, we conclude that most of the former proteins indeed possessed their complete $\mathrm{C}$ termini.

Sequential extraction experiments demonstrated that the endogenous Xenopus enzyme and hAChE-E6 partitioned mainly 

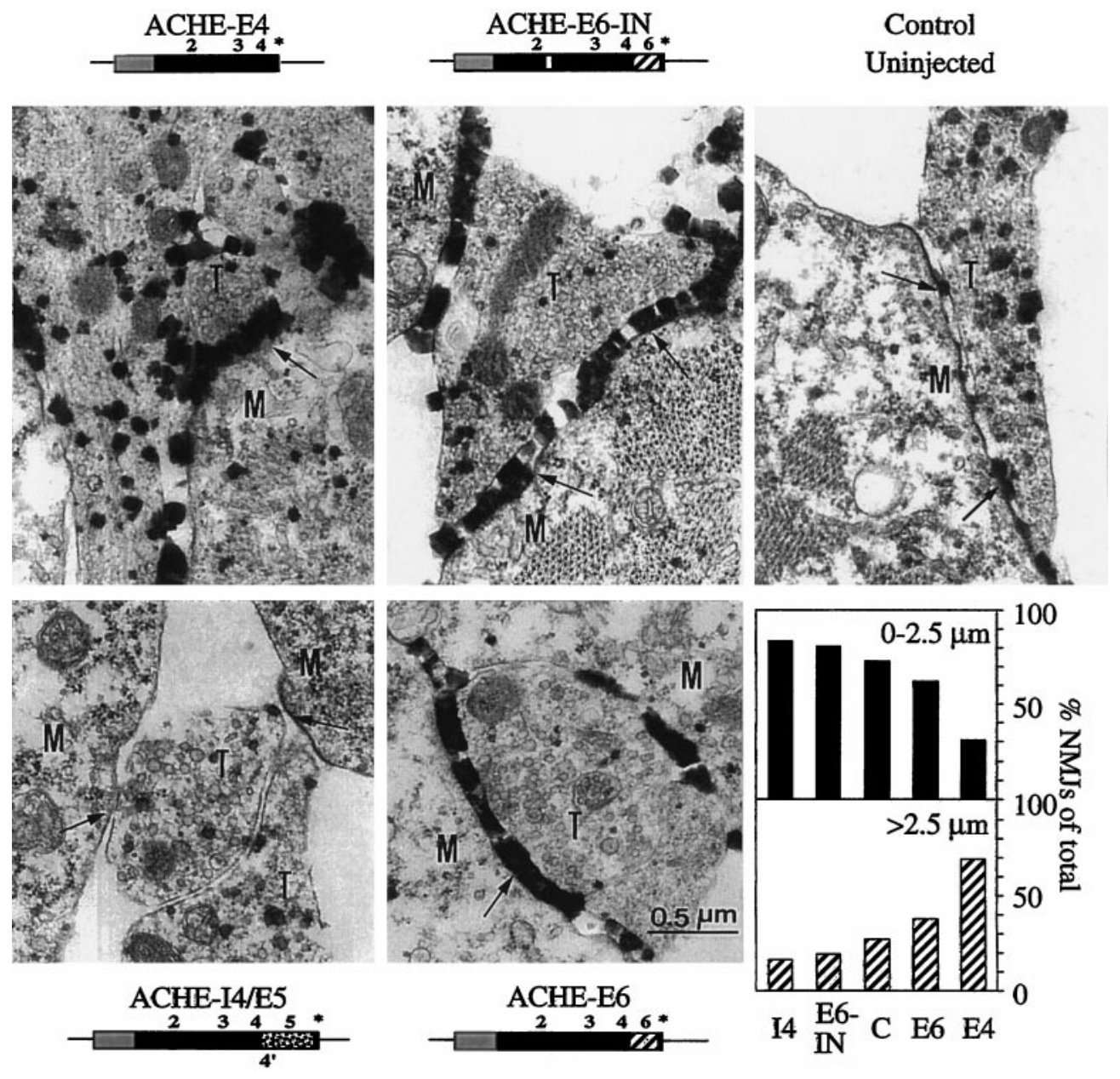
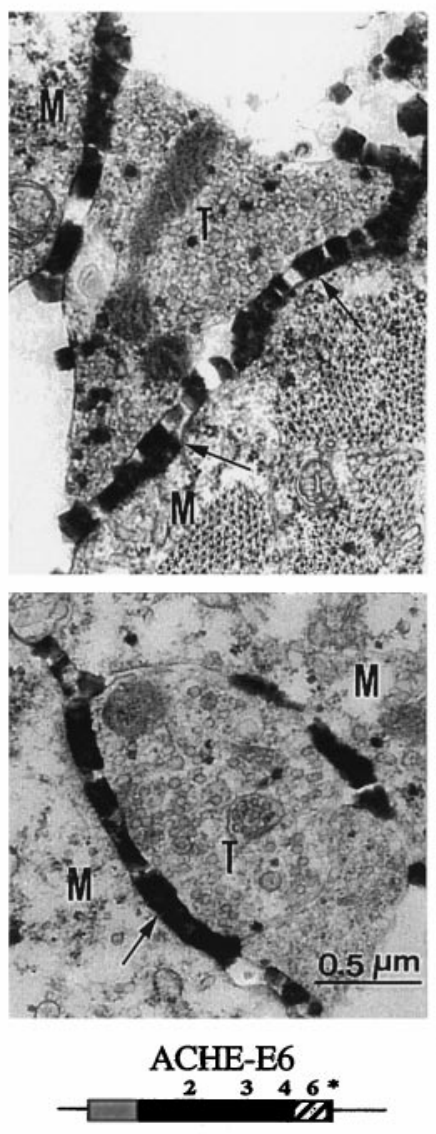

\section{I4}

Figure 5. AChE-E6 and AChE-E4 enhance NMJ length, whereas AChE-I4 and AChE-E6-IN do not. Two-day-old DNA-injected and control uninjected Xenopus embryos were stained for catalytically active $\mathrm{AChE}$ and examined by electron microscopy. Representative images of NMJs from embryos injected with each of the vectors are shown. Note the enhanced staining apparent as dark electron-dense deposits in NMJs from AChE-E6-, AChE-E6-IN-, and AChEE4-injected embryos as compared with controls. $T$, Nerve terminal; $M$, muscle cell; arrows points at synaptic clefts. Bottom right panel, NMJ population analysis. Electron microscope NMJ images $(16,31,43$, and 66 sections from AChEE4, -E6-IN, -I4, and -E6 injected and 55 sections from control uninjected embryos, respectively) were used for postsynaptic length measurements. The percentage of synapses with lengths shorter or longer than $2.5 \mu \mathrm{m}$ are presented for NMJs from embryos injected with each vector. Note that expression of AChE-E6 and AChE-E4 increases postsynaptic length as compared with controls. between the low-salt and the low-salt-detergent fractions, whereas hAChE-E4 and hAChE-I4 were almost completely solubilized in low salt. These observations extend previous findings (Seidman et al., 1995) that unlike hAChE-E6, hAChE-I4 is secreted to the medium by Xenopus embryos and imply that the $\mathrm{C}$ terminus encoded by I4 does not contribute to the membrane interaction properties of this enzyme. Furthermore, these results are in agreement with the well known membrane association of AChE-E6 (for review, see Massoulie et al., 1993). Therefore, of all $\mathrm{AChE}$ forms tested, only proteins containing the E6-derived $\mathrm{C}$ terminus could associate with neuronal membranes and support growth through such association.

The endogenous Xenopus enzyme, which has not yet been extensively characterized or molecularly cloned, displayed hydrodynamic properties and subcellular interactions similar to those of transgenic hAChE-E6 and resembles the synaptic form of mammalian AChE encoded by ACHE-E6. Xenopus AChE is more resistant to heat and to the anticholinesterase echothiophate than the human enzyme (this report) and does not form heteromeric multimers with hAChE (Seidman et al., 1994). However, despite these distinctions, Xenopus neurons and NMJs respond to the morphogenic activities of hAChE in an evolutionarily conserved manner. This emphasizes the importance of these functions and may allude to their early emergence in evolution.

In addition to its extracellular function in the hydrolysis of synaptic ACh, we demonstrate here that when intracellularly expressed, the membrane-associated AChE-E6 protein but not the alternatively spliced AChE-I4-secreted protein promotes autologous neurite extension of Xenopus neurons. Similar neurite growth activity can be induced by mutation-inactivated AChE-E6 but not by the enzymatically active truncated AChE-E4 enzyme. In cultures of chick sympathetic neurons, rat hippocampus, or retinal ganglion cells, $\mathrm{AChE}$ inhibitors interacting with the peripheral site of the enzyme prevented the neurite growth effect and fasciculation exerted by externally added AChE (Layer et al., 1993; Jones et al., 1995; Small et al., 1995). However, the active site organophosphate inhibitor echothiophate, which totally inhibits cholinesterase activity, did not block AChE-induced growth. This indicated that the neurite promotion effects were not caused by enzyme activity per se. However, the effect of these pharmacological agents could be unrelated to their binding to AChE, whereas elevation of the levels of intracellularly produced AChE protein unequivocally establishes a novel noncatalytic function for this protein. Like the process extension in rat glioma cells microinjected with ACHE-E6 DNA (Karpel et al., 1996), the neurite growth-promoting effect was spatially limited to those cells expressing the enzyme and did not extend to adjacent, non-hAChE-expressing neurons. This suggests that it involves the detergent-extractable fraction of AChE-E6 and that it is associated with membrane protein signaling.

How does AChE promote neurite growth? A group of adhesion molecules, such as neurotactin, neuroligin, and gliotactin, contain extracellular domains showing a uniformly distributed homology to cholinesterases. Their cysteine positions correspond 
to those involved in the formation of AChE intramolecular disulfide bonds, suggesting that these adhesion molecules may resume tertiary structure similar to that of cholinesterases. Although none of these proteins is a catalytically active esterase, replacement of the extracellular domain of Drosophila neurotactin with the core domain of $\mathrm{AChE}$ created a chimeric protein promoting cell adhesion (Darboux et al., 1996). Drosophila AChE itself or neurotactin lacking most of its intracellular or extracellular domains failed to do so, suggesting membrane-associated signaling operable with the AChE core domain. Thus, AChE may promote neurite extension by modulating the adhesion capacity of neurites.

$\beta$-Neurexins have been identified as the neuronal membrane partners interacting with neuroligins (Ichtchenko et al., 1995). This indicates that the core AChE domain encoded by exons 2-4 and corresponding to the cholinesterase-like domains of neurotactin and neuroligins could also operate by supporting recognition of neurexins and related ligand(s). In mammals, it has been postulated that association between $\beta$-neurexins and neuroligins contributes to axon growth and cell-cell and cell-extracellular matrix interactions (Puschel and Betz, 1995). Unlike the core polypeptide of AChE, the E6-derived $\mathrm{C}$ terminus does not share homology with the neurotactin family members (Seidman et al., 1995) but could associate the enzyme with the cell membrane. Both hAChE-E6 and hAChE-E6-IN, but not hAChE-E4 or hAChE-I4, could potentially associate with the cell membrane through the E6 $\mathrm{C}$ terminus and interact, through the core $\mathrm{AChE}$ domain, with a $\beta$-neurexin-like ligand expressed on the surface of the same or other cells or associated with the extracellular matrix. This would elicit signal transduction by the intracellular domain of the ligand, which can induce neurite extension. Likewise, the process extension effect exerted by hAChE-E6 on glia (Karpel et al., 1996) can be attributed to interaction with the corresponding ligand of the AChE homologous protein gliotactin (Auld et al., 1995). Moreover, the recent discovery of the novel Neurexin 4 expressed in epithelial cells (Baumgartner et al., 1996) extends this hypothesis also to non-neuronal sites. This theory is strengthened further by our recent observation that transgenic expression of hAChE in mice modulates the production of $\beta$-neurexins in the mouse spinal cord (Andres et al., 1997). Molecular cloning of Xenopus neurexins would be required to further investigate this mechanism.

The novel function of $\mathrm{AChE}$ in promoting neurite growth explains the early developmental involvement for this protein before synaptogenesis, supporting descriptive theories based on its spatiotemporal expression pattern in avian embryogenesis (Layer et al., 1995). It presents an interesting example of multiple, seemingly unrelated functions for one protein. That such functional duality in various tissues may be a more general phenomenon than we are currently aware of is indicated from findings with other proteins, such as lactate dehydrogenase (LDH), which is both a hepatic enzyme (Baumgart et al., 1996) and a structural crystallin protein in the lens (Chiou et al., 1991; Voorter et al., 1993). Although ACh hydrolysis was the first and foremost identified function of $\mathrm{AChE}$, distinct elements on the surface of this protein might have been preserved during evolution because of their interaction capacities with specific diverse molecules, which serve for different cellular functions.

Unlike its neurite growth-promoting activity, our current findings demonstrate that the synaptogenic activity of AChE is tightly related with its catalytic activity yet not dependent on the E6derived $\mathrm{C}$ terminus. Thus the synaptic accumulation of the cata- lytically active, truncated AChE-E4 sufficed to enhance NMJ development in vivo, whereas the inert AChE-E6-IN did not enlarge these synapses, although its structural properties suggest distribution similar to that of the native enzyme. This in turn suggests that the I4-derived $\mathrm{C}$ terminus is actively involved in the exclusion of AChE-I4 from the synaptic cleft and attributes an important role to the effective termination of cholinergic neurotransmission in synapse development. Altogether, one should view $\mathrm{AChE}$ as a modular macromolecule, designed to transduce neurite growth signals as well as synapse development ones by virtue of a concerted combination of its core protein domain, alternative $\mathrm{C}$ termini and its ACh hydrolytic capacity.

\section{REFERENCES}

Anderson MJ, Cohen MW, Zorychta E (1977) Effects of innervation on the distribution of acetylcholine receptors on cultured muscle cells. J Physiol (Lond) 268:731-756.

Andres C, Beeri R, Friedman A, Lev-Lehman E, Henis S, Timberg R, Shani M, Soreq H (1997) Acetylcholinesterase-transgenic mice display embryonic modulations in spinal cord choline acetyltransferase and neurexin $\mathrm{I} \beta$ gene expression followed by late-onset neuromotor degeneration. Proc Natl Acad Sci USA 95:8173-8178.

Auld VJ, Fetter RD, Broadie K, Goodman CS (1995) Gliotactin, a novel transmembrane protein on peripheral glia, is required to form the blood-nerve barrier in Drosophila. Cell 81:757-767.

Baumgart E, Fahimi H, Stich A, Volki A (1996) L-lactate dehydrogenase A4- and A3B isoforms are bona fide peroxisomal enzymes in rat liver. Evidence for involvement in intraperoxisomal NADH reoxidation. J Biol Chem 271:3846-3855.

Baumgartner S, Littleton JT, Broadie K, Bhat MA, Harbecke R, Lengyel JA, Chiquet-Ehrismann R, Prokop A, Bellen HJ (1996) A Drosophila neurexin is required for septate junction and blood-nerve barrier formation and function. Cell 87:1059-1068.

Ben Aziz-Aloya R, Seidman S, Timberg R, Sternfeld M, Zakut H, Soreq $\mathrm{H}$ (1993) Expression of a human acetylcholinesterase promoterreporter construct in developing neuromuscular junctions of Xenopus embryos. Proc Natl Acad Sci USA 90:2471-2475.

Chiou SH, Lee HJ, Huang SM, Chang GG (1991) Kinetic comparison of caiman epsilon-crystallin and authentic lactate dehydrogenases of vertebrates. J Protein Chem 10:161-166.

Darboux I, Barthalay Y, Piovant M, Hipeau-Jaczquotte R (1996) The structure-function relationships in Drosophila neurotactin show that cholinesterasic domains may have adhesive properties. EMBO J 15:4835-4843.

de La Escalera S, Bockamp EO, Moya F, Piovant M, Jimenez F (1990) Characterization and gene cloning of neurotactin, a Drosophila transmembrane protein related to acetylcholinesterase. EMBO J 9:3593-3601.

Deuchar EM (1966) Biochemical aspects of amphibian development. London: Methuen.

Duval N, Massoulie J, Bon S (1992) H and T subunits of acetylcholinesterase from Torpedo, expressed in COS cells generate all types of globular forms. J Cell Biol 118:641-653.

Higuchi R (1990) Recombinant PCR. In: PCR protocols (Innis MA, Gellfand DH, Sninsky JJ, White TJ, eds), pp 177-183. San Diego: Academic.

Ichtchenko K, Hata Y, Nguyen T, Ullrich B, Missler M, Moomaw C, Sudhof TC (1995) Neuroligin 1: a splice site-specific ligand for $\beta$-neurexins. Cell 81:435-443.

Jones SA, Holmes C, Budd TC, Greenfield SA (1995) The effect of acetylcholinesterase on outgrowth of dopaminergic neurons in organotypic slice culture of rat midbrain. Cell Tissue Res 279:323-330.

Karpel R, Ben Aziz-Aloya R, Sternfeld M, Ehrlich G, Ginzberg D, Tarroni P, Clementi F, Zakut H, Soreq H (1994) Expression of three alternative acetylcholinesterase messenger RNAs in human tumor cell lines of different tissue origins. Exp Cell Res 210:268-277.

Karpel R, Sternfeld M, Ginzberg D, Guhl E, Graessman A, Soreq H (1996) Overexpression of alternative human acetylcholinesterase forms modulates process extensions in cultured glioma cells. J Neurochem 66:114-123.

Layer PG, Willbold E (1995) Novel functions of cholinesterases in development, physiology and disease. Prog Histochem Cytochem 29:1-99. 
Layer PG, Weikert T, Alber R (1993) Cholinesterases regulate neurite growth of chick nerve cells in vitro by means of a non-enzymatic mechanism. Cell Tissue Res 273:219-226.

Massoulie J, Pezzementi L, Bon S, Krejci E, Vallette F-M (1993) Molecular and cellular biology of cholinesterases. Prog Neurobiol 41:31-91.

Neville LF, Gnatt A, Padan R, Seidman S, Soreq H (1990) Anionic site interactions in human butyrylcholinesterase disrupted by two adjacent single point mutations. J Biol Chem 265:20735-20738.

Nieuwkoop PD, Faber J (1967) Normal table of Xenopus laevis, Ed 2. Amsterdam: North Holland.

Puschel AW, Betz H (1995) Neurexins are differentially expressed in the embryonic nervous system of mice. J Neurosci 15:2849-2856.

Salpeter M (1967) Electron microscope radioautography as a quantitative tool in enzyme cytochemistry. I. The distribution of acetylcholinesterase at motor endplates of a vertebrate twitch muscle. J Cell Biol 32:379-389.

Schwarz M, Loewenstein-Lichtenstein Y, Glick D, Liao J, NorgaardPedersen B, Soreq H (1995a) Successive organophosphate inhibition and oxime reactivation reveals distinct responses of recombinant human cholinesterase variants. Mol Brain Res 31:101-110.

Schwarz M, Glick D, Loewenstein Y, Soreq H (1995b) Engineering of human cholinesterases explains and predicts diverse consequences of administration of various drugs and poisons. Pharmacol Ther 67:283-322.

Seidman S, Soreq H (1996) Transgenic Xenopus microinjection methods and developmental neurobiology. Neuromethods series (Boulton A, Baker GB, eds), pp 1-198. Totowa, NJ: Humana.

Seidman S, Ben Aziz-Aloya R, Timberg R, Loewenstein Y, Velan B, Shaffeman A, Liao J, Norgaard-Pedersen B, Brodbeck U, Soreq H
(1994) Overexpressed monomeric human acetylcholinesterase induces subtle ultrastructural modifications in developing neuromuscular junctions of Xenopus laevis embryos. J Neurochem 62:1670-1681.

Seidman S, Sternfeld M, Ben Aziz-Aloya R, Timberg R, Kaufer-Nachum D, Soreq H (1995) Synaptic and epidermal accumulation of human acetylcholinesterase are encoded by alternative 3 '-terminal exons. Mol Cell Biol 15:2993-3002.

Small DH, Reed G, Whitefield B, Nurcombe V (1995) Cholinergic regulation of neurite outgrowth from isolated chick sympathetic neurons in culture. J Neurosci 15:144-151.

Soreq H, Ben Aziz R, Prody CA, Seidman S, Gnatt A, Neville L, Lieman-Hurwitz J, Lev-Lehman E, Lapidot-Lifson Y, Zakut H (1990) Molecular cloning and construction of the coding region for human acetylcholinesterase reveals a $\mathrm{G}+\mathrm{C}$-rich attenuating structure. Proc Natl Acad Sci USA 87:9688-9692.

Spitzer NC, Lamborghini JC (1976) The development of the action potential mechanism of amphibian neurons isolated in culture. Proc Natl Acad Sci USA 73:1641-1645.

Sussman JL, Harel M, Frolow F, Oefner C, Goldman A, Toker L, Silman I (1991) Atomic structure of acetylcholinesterase from Torpedo californica: a prototypic acetylcholine-binding protein. Science 253:872-879.

Tabti N, Poo, M-m (1995) Study on the induction of spontaneous transmitter release at early nerve-muscle contacts in Xenopus cultures. Neurosci Lett 173:21-26.

Taylor P, Radic Z (1994) Cholinesterases: from genes to proteins. Annu Rev Pharmacol Toxicol 34:281-320.

Voorter CE, Wintjes LT, Heinstra PW, Bloemendal H, De-Jong WW (1993) Comparison of stability properties of lactate dehydrogenase B4/ epsilon-crystalline from different species. Eur J Biochem 211:643-648. 